\title{
Nonabelian dark matter with resonant annihilation
}

\author{
Cheng-Wei Chiang, ${ }^{a, b, c}$ Takaaki Nomura $^{a, d}$ and Jusak Tandean ${ }^{e}$ \\ ${ }^{a}$ Department of Physics and Center for Mathematics and Theoretical Physics, \\ National Central University, Chungli 320, Taiwan \\ ${ }^{b}$ Institute of Physics, Academia Sinica, \\ Taipei 115, Taiwan \\ ${ }^{c}$ Physics Division, National Center for Theoretical Sciences, \\ Hsinchu 300, Taiwan \\ ${ }^{d}$ Department of Physics, National Cheng-Kung University, \\ Tainan 701, Taiwan \\ ${ }^{e}$ Department of Physics and Center for Theoretical Sciences, \\ National Taiwan University, Taipei 106, Taiwan \\ E-mail: chengwei@phy.ncu.edu.tw, nomura@mail.ncku.edu.tw, \\ jtandean@yahoo.com
}

ABSTRACT: We construct a model based on an extra gauge symmetry, $\mathrm{SU}(2)_{X} \times \mathrm{U}(1)_{B-L}$, which can provide gauge bosons to serve as weakly-interacting massive particle dark matter. The stability of the dark matter is naturally guaranteed by a discrete $Z_{2}$ symmetry that is a subgroup of $\mathrm{SU}(2)_{X}$. The dark matter interacts with standard model fermions by exchanging gauge bosons which are linear combinations of $\mathrm{SU}(2)_{X} \times \mathrm{U}(1)_{B-L}$ gauge bosons. With the appropriate choice of representation for the new scalar multiplet whose vacuum expectation value spontaneously breaks the $\mathrm{SU}(2)_{X}$ symmetry, the relation between the new gauge boson masses can naturally lead to resonant pair annihilation of the dark matter. After exploring the parameter space of the new gauge couplings subject to constraints from collider data and the observed relic density, we use the results to evaluate the cross section of the dark matter scattering off nucleons and compare it with data from the latest direct detection experiments. We find allowed parameter regions that can be probed by future direct searches for dark matter and LHC searches for new particles.

KeYwords: Phenomenological Models

ARXIV EPRINT: 1306.0882 


\section{Contents}

1 Introduction 1

2 A model of dark massive gauge boson $\quad 2$

3 Constraints from collider experiments 5

4 Resonant dark matter annihilation and relic density $\quad 7$

$\begin{array}{lll}5 & \text { Direct detection of dark matter } & 8\end{array}$

$\begin{array}{llr}6 & \text { Comments on collider phenomenology } & 9\end{array}$

$\begin{array}{lll}7 & \text { Summary and discussion } & 12\end{array}$

$\begin{array}{ll}\text { A Feynman rules for new gauge interactions } & 13\end{array}$

B Kinetic mixing between $\mathrm{U}(1)_{Y}$ and $\mathrm{U}(1)_{B-L} \quad 14$

\section{Introduction}

The standard model (SM) of particle physics has been very successful in describing an enormous amount of experimental data at energies up to $\mathcal{O}(100) \mathrm{GeV}$. There are, however, questions remaining that require physics beyond the minimal SM to address. Among the outstanding issues are the explanations for the astronomical evidence of dark matter $(\mathrm{DM})$ and for the numerous experimental indications of neutrino mass [1]. It is then of great interest to explore a new physics scenario in which the DM and neutrino sectors are intimately connected.

Previously, we have considered a simple model which provides not only DM of the popular weakly-interacting massive particle (WIMP) type, but also a means to endow neutrinos with mass [2]. The DM candidate belongs to a complex scalar singlet stabilized by a $Z_{2}$ symmetry that is not imposed in an ad hoc way, but instead emerges from an extra Abelian gauge group related to baryon number minus lepton number, $\mathrm{U}(1)_{B-L}$, that is spontaneously broken by the nonzero vacuum expectation value (VEV) of a new scalar field, in the Krauss-Wilczek manner [3]. Light neutrino masses are produced via the wellknown seesaw mechanism [4-11], which is triggered with the involvement of the same new scalar field after the addition of right-handed neutrinos. The DM relic density receives contributions mainly from diagrams mediated by the Higgs boson and also those mediated by the $\mathrm{U}(1)_{B-L}$ gauge boson, $Z^{\prime}$. It turns out that constraints from collider data and the observed relic density together imply that the $Z^{\prime}$ mass has to be in the resonance region of the $Z^{\prime}$-mediated DM annihilation, namely about twice the DM mass. Furthermore, results from DM direct detection experiments and Higgs data from the LHC favor the dominance 
of the $Z^{\prime}$-exchange contributions to the relic density. All this motivates us to look for a different possible scenario in which the resonance condition can be fulfilled naturally.

In this paper, we demonstrate that such a possibility can be realized in a model where the role of WIMP DM is played by massive gauge bosons associated with a nonabelian symmetry. Although most of the WIMP DM candidates proposed in the literature are either fermionic or spinless, those with spin one have also been considered before [12-20]. Here we construct a model based on the gauge group $\mathrm{G}_{\mathrm{SM}} \times \mathrm{SU}(2)_{X} \times \mathrm{U}(1)_{B-L}$, where $\mathrm{G}_{\mathrm{SM}}$ refers to the SM group and the extra gauge symmetries offer gauge bosons which can act as WIMP candidates. The stability of the DM is naturally maintained by a discrete $Z_{2}$ symmetry which is a subgroup of the new nonabelian gauge group, $\mathrm{SU}(2)_{X}$. This $Z_{2}$ symmetry appears after the spontaneous breaking of $\mathrm{SU}(2)_{X}$ by the nonzero VEV of a new scalar multiplet, following the Krauss-Wilczek mechanism [3]. Then the $Z_{2}$-odd gauge bosons associated with $\mathrm{SU}(2)_{X}$ can serve as DM if they are lighter than other $Z_{2}$-odd particles in the model. Since SM fermions are charged under $\mathrm{U}(1)_{B-L}$, the DM can interact with SM fermions at tree level by exchanging gauge bosons which are obtained from the linear combinations of $\mathrm{SU}(2)_{X}$ and $\mathrm{U}(1)_{B-L}$ gauge fields. Thus, the new gauge interactions are responsible for both the relic abundance and the DM interactions with nucleons. Another interesting feature of the model is that, the $\mathrm{DM}$ being made up of $\mathrm{SU}(2)_{X}$ gauge bosons, its mass is related to the masses of the mediating gauge bosons, implying that resonant pair annihilation can be naturally achieved by choosing suitable representations of the scalar fields involved in the breaking of the $\mathrm{SU}(2)_{X} \times \mathrm{U}(1)_{B-L}$ gauge symmetry and ensuring that their VEVs are sufficiently well separated. What's more, the presence of the $\mathrm{U}(1)_{B-L}$ gauge symmetry requires the introduction of right-handed neutrinos for gauge-anomaly cancellation, which in turn participate in the type-I seesaw mechanism to generate light neutrino masses [4-11], with the right-handed neutrino masses being connected to the $\mathrm{U}(1)_{B-L}$ breaking scale. This model turns out to have sufficient parameter space that is consistent with current collider, relic density, and DM direct search data. Therefore, it can be probed further by ongoing or future DM direct detection experiments, and some of the new particles may be observable at the LHC with sufficient luminosities.

This paper is organized as follows. The next section contains the details of our model which possesses WIMP DM composed of the gauge bosons of an extra nonabelian gauge symmetry. We explain how the choices of the new particles and their quantum numbers can naturally translate into resonant annihilation of the DM. In section 3, we examine constraints on the new gauge couplings from collider data. In section 4 , we deal with the relic density of our DM candidates and extract the parameter values allowed by its observed value. In section 5 , we use the results to predict the DM-nucleon scattering cross-section and compare it with current data from direct detection experiments. In section 6 , we comment on the collider phenomenology of the new particles in our model. We conclude in section 7 with the summary of our study and some more discussion.

\section{A model of dark massive gauge boson}

Compared to the SM, the new model contains the additional gauge group $\mathrm{SU}(2)_{X} \times \mathrm{U}(1)_{B-L}$, where $X$ refers to the massive gauge boson that serves as the DM, 


\begin{tabular}{|c|c|c|c|c|c|c|c|c|c|c|c|c|c|}
\hline & $f_{\mathrm{SM}}$ & $\nu_{R}$ & $H$ & $S$ & $\phi_{2}$ & $\phi_{1}$ & $\phi_{0}$ & $\phi_{-1}$ & $\phi_{-2}$ & $X$ & $X^{\dagger}$ & $C_{3}$ & $E$ \\
\hline $\mathrm{SU}(2)_{X}\left[\mathrm{U}(1)_{B-L}\right]$ & $1[B-L]$ & $1[-1]$ & $1[0]$ & $1[2]$ & $5[2]$ & $5[2]$ & $5[2]$ & $5[2]$ & $5[2]$ & $3[0]$ & $3[0]$ & $3[0]$ & $1[0]$ \\
\hline$T_{3 X}$ & 0 & 0 & 0 & 0 & 2 & 1 & 0 & -1 & -2 & 1 & -1 & 0 & 0 \\
\hline$Z_{2}^{X}$ & + & + & + & + & + & - & + & - & + & - & - & + & + \\
\hline
\end{tabular}

Table 1. The charge assignments under $\mathrm{SU}(2)_{X} \times \mathrm{U}(1)_{B-L}$ and $Z_{2}^{X}$ parity of the fermions, scalars and new gauge bosons in the model, with $f_{\mathrm{SM}}$ referring to SM fermions, $X=\left(C_{1}-i C_{2}\right) / \sqrt{2}$, and $T_{3 X}$ denoting the eigenvalue of the third generator of $\mathrm{SU}(2)_{X}$.

whereas $B$ and $L$ stand for baryon and lepton numbers, respectively. We denote the gauge fields associated with $\mathrm{SU}(2)_{X}$ and $\mathrm{U}(1)_{B-L}$ by $C_{k}^{\mu}$ and $E^{\mu}$, respectively, $k=1,2,3$, and their coupling constants $g_{X}$ and $g_{B-L}$. The model also has new complex scalar fields $S$ and $\Phi_{5}$ as well as three extra fermions $\nu_{k R}$, all of which are singlets under the SM gauge group, but carry nonzero $\mathrm{U}(1)_{B-L}$ charges. Under $\mathrm{SU}(2)_{X}$ transformations, $S$ is a singlet, while $\Phi_{5}$ is a five-plet represented by the column matrix $\Phi_{5}=\left(\phi_{2}, \phi_{1}, \phi_{0}, \phi_{-1}, \phi_{-2}\right)^{\mathrm{T}}$, where $\phi_{a}$ corresponds to the eigenvalue $T_{3 X}=a$ of the third generator of $\mathrm{SU}(2)_{X}$. In table 1 we collect the $\mathrm{SU}(2)_{X} \times \mathrm{U}(1)_{B-L}$ quantum number assignments for the fermions, scalars, and new gauge bosons in the model, with $H$ being the usual scalar doublet.

The renormalizable Lagrangian for $S$ and $\Phi_{5}$, with $H$ included in the potential $\mathcal{V}$, is

$$
\mathcal{L}=\left(\mathcal{D}^{\mu} S\right)^{\dagger} \mathcal{D}_{\mu} S+\left(\mathcal{D}^{\mu} \Phi_{5}\right)^{\dagger} \mathcal{D}_{\mu} \Phi_{5}-\mathcal{V}
$$

where

$$
\begin{aligned}
\mathcal{D}^{\mu} S= & \partial^{\mu} S+2 i g_{B-L} E^{\mu} S, \\
\mathcal{D}^{\mu} \Phi_{5}= & \partial^{\mu} \Phi_{5}+i g_{X} C_{k}^{\mu} \mathcal{T}_{k}^{(5)} \Phi_{5}+i g_{B-L} E^{\mu} \mathcal{Q}_{B-L}^{(5)} \Phi_{5}, \\
\mathcal{V}= & -\mu_{\Phi}^{2} \Phi_{5}^{\dagger} \Phi_{5}+\left(\lambda_{S}|S|^{2}-\mu_{S}^{2}\right)|S|^{2}+\left(\lambda_{H} H^{\dagger} H-\mu_{H}^{2}\right) H^{\dagger} H \\
& + \text { (other quartic terms) } .
\end{aligned}
$$

In $\mathcal{D}^{\mu} \Phi_{5}$ above, summation over $k=1,2,3$ is implicit, and $\mathcal{T}_{k}^{(5)}$ and $\mathcal{Q}_{B-L}^{(5)}$ are matrices for the generators of $\mathrm{SU}(2)_{X}$ and $\mathrm{U}(1)_{B-L}$, respectively, acting on $\Phi_{5}$, where

$$
\begin{array}{lc}
\mathcal{T}_{1}^{(5)}=\frac{1}{2}\left(\begin{array}{ccccc}
0 & 2 & 0 & 0 & 0 \\
2 & 0 & \sqrt{6} & 0 & 0 \\
0 & \sqrt{6} & 0 & \sqrt{6} & 0 \\
0 & 0 & \sqrt{6} & 0 & 2 \\
0 & 0 & 0 & 2 & 0
\end{array}\right), & \mathcal{T}_{2}^{(5)}=\frac{i}{2}\left(\begin{array}{ccccc}
0 & -2 & 0 & 0 & 0 \\
2 & 0 & -\sqrt{6} & 0 & 0 \\
0 & \sqrt{6} & 0 & -\sqrt{6} & 0 \\
0 & 0 & \sqrt{6} & 0 & -2 \\
0 & 0 & 0 & 2 & 0
\end{array}\right), \\
\mathcal{T}_{3}^{(5)}=\operatorname{diag}(2,1,0,-1,-2), & \mathcal{Q}_{B-L}^{(5)}=\operatorname{diag}(2,2,2,2,2) .
\end{array}
$$

In this paper, we consider the scenario in which the $\mathrm{SU}(2)_{X} \times \mathrm{U}(1)_{B-L}$ gauge symmetry is spontaneously broken according to

$$
\mathrm{SU}(2)_{X} \times \mathrm{U}(1)_{B-L} \stackrel{\langle S\rangle}{\longrightarrow} \mathrm{SU}(2)_{X} \times Z_{2}^{B-L} \stackrel{\left\langle\Phi_{5}\right\rangle}{\longrightarrow} Z_{2}^{X} \times Z_{2}^{B-L},
$$


where $\langle S\rangle=v_{S} / \sqrt{2}$ and $\left\langle\Phi_{5}\right\rangle=\left(v_{\Phi}, 0,0,0,0\right)^{\mathrm{T}} / \sqrt{2}$ are the VEVs of $S$ and $\Phi_{5}$, with $v_{S} \gg v_{\Phi}>0$. Since $\left\langle\Phi_{5}\right\rangle \neq 0$ occurs via its $T_{3 X}=2$ component, $\left\langle\phi_{2}\right\rangle \neq 0$, the $Z_{2}^{X}$ symmetry emerges naturally as a subgroup of $\mathrm{SU}(2)_{X}$ and the particles with even (odd) $T_{3 X}$ values will be $Z_{2}^{X}$ even (odd), as table 1 shows. On the other hand, $Z_{2}^{B-L}$ is the remnant of $\mathrm{U}(1)_{B-L}$ after $\langle S\rangle \neq 0$, as discussed in ref. [2], but does not play a role in the stabilization of $X$. Thus, in this scenario the remaining $Z_{2}^{X}$ guarantees the stability of the lightest $Z_{2}^{X}$-odd particle(s), which can therefore act as DM. Here we choose the gauge boson $X=\left(C_{1}-i C_{2}\right) / \sqrt{2}$ and its conjugate $X^{\dagger}$ to be the DM, hence tacitly taking the $Z_{2}^{X}$-odd scalar bosons to be more massive than $X$. It is worth mentioning that we would arrive at the same results below if $\left\langle\Phi_{5}\right\rangle \neq 0$ through its $T_{3 X}=-2$ component instead. As for $H$, its VEV is also nonvanishing and breaks the electroweak symmetry just as in the SM. We assume that the other parameters in the potential $\mathcal{V}$ are such that the vacuum has the above desired properties, leaving a detailed analysis of $\mathcal{V}$ for future work.

After $\mathrm{SU}(2)_{X} \times \mathrm{U}(1)_{B-L}$ spontaneously breaks into $Z_{2}^{X} \times Z_{2}^{B-L}$, the new gauge bosons acquire in $\mathcal{L}$ the mass terms

$$
\begin{aligned}
\mathcal{L}_{\mathrm{m}}= & \left\langle\Phi_{5}^{\dagger}\right\rangle\left[g_{X} C_{k}^{\mu} \mathcal{T}_{k}^{(5)}+g_{B-L} E^{\mu} \mathcal{Q}_{B-L}^{(5)}\right]\left[g_{X} C_{k^{\prime} \mu} \mathcal{T}_{k^{\prime}}^{(5)}+g_{B-L} E_{\mu} \mathcal{Q}_{B-L}^{(5)}\right]\left\langle\Phi_{5}\right\rangle \\
& +4 g_{B-L}^{2} E^{2}\langle S\rangle^{2} \\
= & g_{X}^{2} v_{\Phi}^{2} X_{\mu}^{\dagger} X^{\mu}+\frac{1}{2}\left(\begin{array}{ll}
C_{3}^{\mu} & E^{\mu}
\end{array}\right)\left(\begin{array}{cc}
4 g_{X}^{2} v_{\Phi}^{2} & 4 g_{X} g_{B-L} v_{\Phi}^{2} \\
4 g_{X} g_{B-L} v_{\Phi}^{2} & 4 g_{B-L}^{2}\left(v_{\Phi}^{2}+v_{S}^{2}\right)
\end{array}\right)\left(\begin{array}{c}
C_{3 \mu} \\
E_{\mu}
\end{array}\right) .
\end{aligned}
$$

From the last line, upon diagonalizing the $2 \times 2$ matrix in the second term, we obtain the eigenvalues

$$
\begin{aligned}
m_{X}^{2}= & g_{X}^{2} v_{\Phi}^{2} \\
m_{Z_{L}, Z_{H}}^{2}= & 2 g_{X}^{2} v_{\Phi}^{2}+2 g_{B-L}^{2}\left(v_{\Phi}^{2}+v_{S}^{2}\right) \\
& \mp 2 \sqrt{\left[g_{X}^{2} v_{\Phi}^{2}-g_{B-L}^{2}\left(v_{\Phi}^{2}+v_{S}^{2}\right)\right]^{2}+4 g_{X}^{2} g_{B-L}^{2} v_{\Phi}^{4}}
\end{aligned}
$$

assuming that $m_{Z_{L}}<m_{Z_{H}}$ for the mass eigenstates $Z_{L}$ and $Z_{H}$ which are given by

$$
\begin{aligned}
\left(\begin{array}{c}
Z_{L} \\
Z_{H}
\end{array}\right) & =\left(\begin{array}{cc}
\cos \theta & \sin \theta \\
-\sin \theta & \cos \theta
\end{array}\right)\left(\begin{array}{c}
C_{3} \\
E
\end{array}\right), \\
\tan (2 \theta) & =\frac{2 g_{X} g_{B-L} R_{v}}{g_{X}^{2} R_{v}-g_{B-L}^{2}\left(1+R_{v}\right)}, \quad R_{v}=\frac{v_{\Phi}^{2}}{v_{S}^{2}} .
\end{aligned}
$$

In this study, we focus on the case in which $v_{S}^{2} \gg v_{\Phi}^{2}$ and $g_{X} \sim g_{B-L}$, implying that

$$
\begin{aligned}
|\theta| & \simeq \frac{g_{X}}{g_{B-L}} R_{v}, \\
m_{Z_{L}}^{2} & \simeq 4 m_{X}^{2}\left(1-R_{v}\right), \\
m_{Z_{H}}^{2} & \simeq 4 m_{X}^{2} \frac{g_{B-L}^{2}}{g_{X}^{2} R_{v}}\left(1+R_{v}\right) .
\end{aligned}
$$


Accordingly, with $R_{v} \ll 1$, we obtain the mass relation

$$
m_{Z_{L}} \simeq 2 m_{X}
$$

which naturally leads to resonant annihilation of the DM pair via the $Z_{L^{-}}$ mediated contribution.

It is worth noting that the five-plet $\Phi_{5}$ is the minimal choice of $\mathrm{SU}(2)_{X}$ representation that can result in the resonant relation in eq. (2.14). In general, for an $\mathrm{SU}(2)_{X}$ isospin value $T_{X}$ and its third component $T_{3 X}$, one would get $m_{X}^{2} / m_{Z_{L}}^{2} \simeq\left[T_{X}\left(T_{X}+1\right)-T_{3 X}^{2}\right] /\left(2 T_{3 X}^{2}\right)$ assuming small mixing angle $\theta$, in analogy to the $\rho$ parameter in the electroweak sector [1].

The neutrino mass-generating sector is the same as that given in ref. [2], the relevant Lagrangian having the form

$$
\mathcal{L}_{m_{\nu}}=i \lambda_{k l} \bar{\nu}_{k R} H^{\mathrm{T}} \tau_{2} L_{l L}-\frac{1}{2} \lambda_{k l}^{\prime} \bar{\nu}_{k R}\left(\nu_{l R}\right)^{\mathrm{c}} S^{\dagger}+\text { H.c. },
$$

where summation over $k, l=1,2,3$ is implicit, $\lambda_{k l}^{(\prime)}$ are free parameters, $\tau_{2}$ is the second Pauli matrix, $L_{l L}$ represents a lepton doublet, and the superscript c indicates charge conjugation. The Dirac and Majorana mass matrices from these terms are $\mathcal{M}_{D}=\lambda v_{H} / \sqrt{2}$ and $\mathcal{M}_{\nu_{R}}=\lambda^{\prime} v_{S} / \sqrt{2}$, respectively, where $v_{H}$ is the VEV of $H$. Hence $v_{S}$ sets the mass scale of the right-handed neutrinos, $\nu_{k R}$. In our examples later on, we will see what values of $v_{S}$ are compatible with the observed relic density and collider data.

Since $X$ is our chosen candidate for DM and interacts with SM fermions by exchanging the $Z_{L, H}$ bosons at tree level, in the following two sections we evaluate the new gauge couplings subject to collider and relic density data. Subsequently, we use the allowed values of the couplings to predict the cross section of the DM-nucleon scattering and compare it with the existing results of DM direct detection experiments.

\section{Constraints from collider experiments}

The gauge bosons $Z_{L}$ and $Z_{H}$ interact with SM fermions at tree level with coupling constants $g_{B-L} \sin \theta$ and $g_{B-L} \cos \theta$, respectively, according to the Feynman rules listed in appendix A. It follows that measurements on processes mediated by $Z_{L}$ and $Z_{H}$ can offer constraints on these couplings. Significant restrictions may be available from the data on $e^{+} e^{-}$and hadron collisions into fermion pairs, which we treat in this section.

We first look at the constraints from $e^{+} e^{-} \rightarrow f \bar{f}$ scattering. In this work we assume that mixing between the $Z$ boson and $Z_{L, H}$ is negligible, but we will comment on the impact of kinetic mixing between them later on and discuss it further in appendix B. In the absence of the mixing, the new gauge couplings have no effects on the $Z$-pole observables at leading order. On the other hand, the measurements of $e^{+} e^{-} \rightarrow f \bar{f}$ at LEP II with center-of-mass energies from 130 to $207 \mathrm{GeV}$ are relevant [21]. We employ the data on the cross section and forward-backward asymmetry for $f=\mu, \tau$ and on the cross section for $f=$ quark. To evaluate the limits on the new couplings, we include both the $Z_{L}$ and $Z_{H}$ contributions to the scattering amplitude, their couplings and masses satisfying the relations in eqs. (2.11)(2.13). Although $Z_{H}$ is much heavier than $Z_{L}$, the fermionic couplings of the latter can 

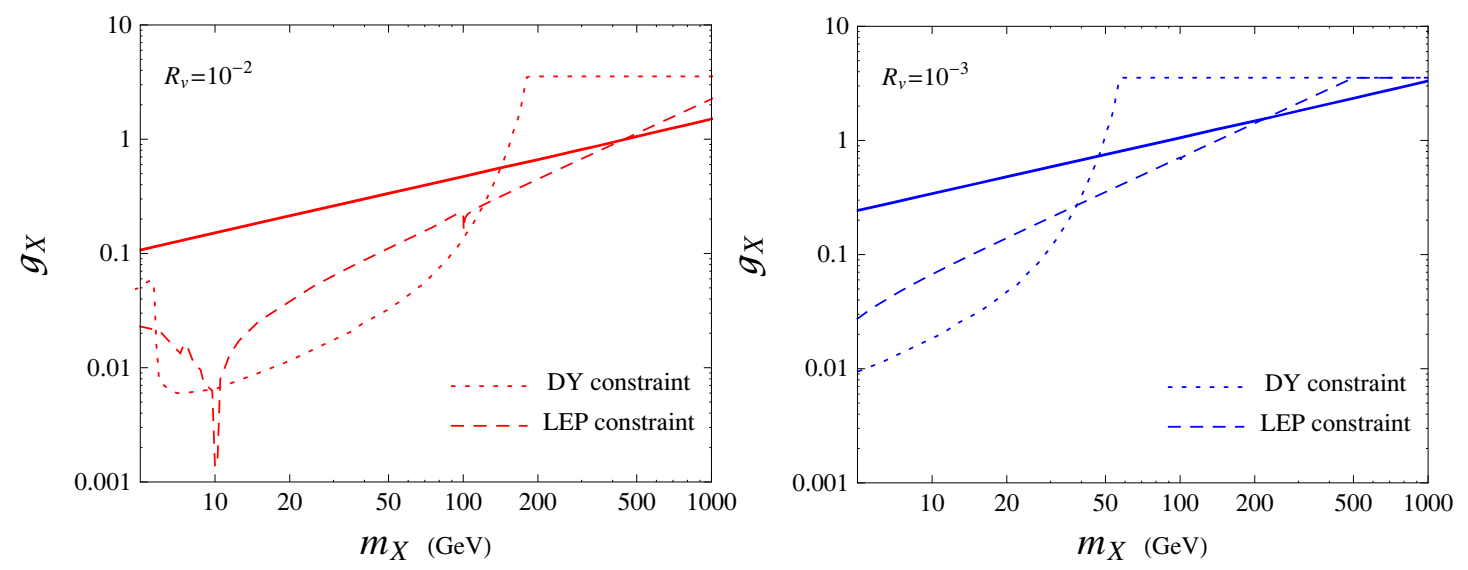

Figure 1. Upper limits on $g_{X}$ versus $m_{X}$ from LEP II and LHC data on $e^{+} e^{-} \rightarrow f \bar{f}$ and Drell-Yan scattering, respectively, for $R_{v}=10^{-2}$ (left) and $10^{-3}$ (right) under the assumption that $g_{X}=g_{B-L}$, compared to the corresponding values of $g_{X}$ (solid curves) consistent with the observed relic density. The horizontal, straight portions of the dashed and dotted curves correspond to the perturbativity condition, $g_{X}<\sqrt{4 \pi}$.

be much smaller than those of the former to compensate for the suppression of the $Z_{H}$ contribution to the amplitude due to its bigger mass. In the examples presented below, the $Z_{H}$ contributions to $e^{+} e^{-} \rightarrow f \bar{f}$ turn out to dominate the $Z_{L}$ ones.

For definiteness and simplicity, hereafter we set $g_{X}=g_{B-L} \geq 0$. Adopting the $90 \%$ confidence-level (CL) ranges of the LEP II measurements [21] and using the formulas given in ref. [22], but with $s$-dependent $Z$ and $Z_{L, H}$ widths [21], we then scan the $m_{X}$ and $R_{v}$ space. To illustrate the results, we display in figure 1 the upper limits on $g_{X}$ versus $m_{X}$ for $R_{v}=10^{-2}$ (red dashed curve) and $10^{-3}$ (blue dashed curve) on the left and right sides, respectively. The horizontal, straight portions of the curves correspond to the perturbativity requirement, $g_{X}<\sqrt{4 \pi}$.

The most recent data from the LHC on the cross-section of the Drell-Yan (DY) process in proton-proton collisions at $\sqrt{s}=7 \mathrm{TeV}$ with $4.5 \mathrm{fb}^{-1}$ of integrated luminosity have revealed no discrepancy from the SM expectations and therefore no evidence of $Z_{L, H}$ bosons [23]. Consequently, we follow the same analysis as in refs. $[2,24]$ to derive upper bounds on the coupling constants using the SM cross-section. In the present case, we can consider the $Z_{L}$ and $Z_{H}$ contributions separately because we focus on events with dilepton invariant mass around $m_{Z_{L}}$ or $m_{Z_{H}}$ where their effects are of different orders for small $R_{v}$. Thus respective constraints are obtained for the pairs $\left(m_{Z_{L}}, g_{B-L} \sin \theta\right)$ and $\left(m_{Z_{H}}, g_{B-L} \cos \theta\right)$. To estimate the DY cross-section numerically, we utilize the CalcHEP package [25] by incorporating the new particles and Feynman rules of our model. Then we apply the one-bin $\log$ likelihood $L L=2[N \ln (N / \nu)+\nu-N]$, where $N(\nu)$ is the number of events predicted by the SM (SM plus the $Z_{L}$ or $Z_{H}$ boson) in the $\ell^{+} \ell^{-}$invariant mass window of $\pm 20 \%$ around the expected $Z_{L}$ or $Z_{H}$ mass, with $\sqrt{s}=7 \mathrm{TeV}$ and $4.5 \mathrm{fb}^{-1}$ of luminosity. The upper limit on the cross-section is obtained from the solved value of $\nu$ for each $Z_{L}$ or $Z_{H}$ mass, after adopting $L L=2.7$ which corresponds to the $90 \%$ CL.

We find that the $Z_{H}$ contribution to the DY process yields a stricter bound on $g_{B-L}$ 
as a function of $m_{Z_{H}}$, as the $Z_{L}$ contribution is strongly suppressed by the small $|\theta|$. We show the resulting upper-limits on $g_{X}\left(=g_{B-L}\right)$ in figure 1 , where $m_{X}$ is related to $m_{Z_{H}}$ by eq. (2.13), for $R_{v}=10^{-2}$ (red dotted curve) and $10^{-3}$ (blue dotted curve) on the left and right, respectively. We notice that the limit in the $R_{v}=10^{-2}$ case becomes large at $m_{X} \sim 5 \mathrm{GeV}$ corresponding to $m_{Z_{H}} \sim m_{Z}$ where the SM background is large.

\section{Resonant dark matter annihilation and relic density}

Now we estimate the relic density of the DM particle, $X$, in order to search for the model parameter space consistent with the observed relic density. The thermal relic abundance is found by solving the Boltzmann equation which describes the number density of the DM. We employ the approximate solution to the Boltzmann equation for the present-day relic density $\Omega$, given by $[26,27]^{1}$

$$
\begin{aligned}
\Omega h^{2} & =\frac{1.07 \times 10^{9}}{\sqrt{g_{*}} m_{\mathrm{Pl}} J \mathrm{GeV}}, \quad J=\int_{x_{f}}^{\infty} d x \frac{\langle\sigma v\rangle}{x^{2}}, \\
x_{f} & =\ln \frac{0.038 g m_{X} m_{\mathrm{Pl}}\langle\sigma v\rangle}{\sqrt{g_{*} x_{f}}},
\end{aligned}
$$

where $h$ denotes the Hubble constant in units of $100 \mathrm{~km} / \mathrm{s} / \mathrm{Mpc}, g_{*}$ is the number of relativistic degrees of freedom below the freeze-out temperature $T_{f}=m_{X} / x_{f}, m_{\mathrm{Pl}}=$ $1.22 \times 10^{19} \mathrm{GeV}$ is the Planck mass, $g=3$ to account for $X$ having spin-1, and $\langle\sigma v\rangle$ is the thermal average of the DM annihilation cross-section. More explicitly [28],

$$
\langle\sigma v\rangle=\frac{x}{8 m_{X}^{5} K_{2}^{2}(x)} \int_{4 m_{X}^{2}}^{\infty} d s \sqrt{s}\left(s-4 m_{X}^{2}\right) K_{1}\left(\sqrt{s} x / m_{X}\right) \sigma_{\mathrm{ann}},
$$

where $K_{i}$ is the modified Bessel function of the second kind of order $i$ and $\sigma_{\text {ann }}$ represents the cross section of $X^{\dagger} X$ annihilation into all possible final states.

Under the assumptions made in section 2, we find that the main contributions to $\sigma_{\text {ann }}$ come from the $s$-channel transitions $X^{\dagger} X \rightarrow Z_{L}^{*} \rightarrow f_{\mathrm{SM}} \bar{f}_{\mathrm{SM}}$. Although $Z_{H}$-mediated diagrams also contribute, in this case they can be neglected because of the suppression due to $m_{Z_{H}} \gg m_{Z_{L}}$ and their lack of the resonance enhancement of the $Z_{L}$-mediated diagrams in the nonrelativistic region $\sqrt{s} \sim 2 m_{X}$ due to $m_{Z_{L}} \simeq 2 m_{X}$. Thus, with the Feynman rules in the appendix $\mathrm{A}$, we arrive at

$$
\begin{aligned}
\sigma_{\mathrm{ann}}=\frac{g_{X}^{2} g_{B-L}^{2} \cos ^{2} \theta \sin ^{2} \theta}{432 \pi} \sum_{f} & \frac{\sqrt{\left(s-4 m_{X}^{2}\right)\left(s-4 m_{f}^{2}\right)}}{m_{X}^{4} s} \frac{s^{2}+20 m_{X}^{2} s+12 m_{X}^{4}}{\left(s-m_{Z_{L}}^{2}\right)^{2}+\Gamma_{Z_{L}}^{2} m_{Z_{L}}^{2}} \\
& \times\left[\left(s+2 m_{f}^{2}\right)\left|\hat{V}_{f}^{Z_{L}}\right|^{2}+\left(s-4 m_{f}^{2}\right)\left|\hat{A}_{f}^{Z_{L}}\right|^{2}\right] N_{\mathrm{c}}^{f},
\end{aligned}
$$

where the sum is over all fermions with masses $m_{f}<m_{X}$ and color factors $N_{\mathrm{c}}^{f}$, the couplings $\hat{V}_{f}^{Z_{L}}$ and $\hat{A}_{f}^{Z_{L}}$ are given in eq. (A.2), and $\Gamma_{Z_{L}}$ is the width of $Z_{L}$. Now, since

\footnotetext{
${ }^{1}$ For a more accurate approximation, see [48].
} 
$m_{Z_{L}}^{2}=4 m_{X}^{2}\left(1-R_{v}\right)$ and $s \geq 4 m_{X}^{2}$ according to eqs. (2.12) and (4.2), respectively, in the denominator of $\sigma_{\text {ann }}$ above we have $\left(s-m_{Z_{L}}^{2}\right)^{2} \geq 16 m_{X}^{4} R_{v}^{2}$. From the collider bounds on $g_{B-L}=g_{X}$ derived in the previous section, we find that for the mass range of interest $16 m_{X}^{4} R_{v}^{2} \gg \Gamma_{Z_{L}}^{2} m_{Z_{L}}^{2}$. Consequently, the $\Gamma_{Z_{L}}$ term can be neglected in the calculation of eq. (4.2).

With eqs. (4.1)-(4.3), we can extract the $\left(g_{X}, m_{X}\right)$ regions compatible with the observed $\Omega$. Its most recent value has been determined by the Planck Collaboration from the Planck measurement and other data to be $\Omega h^{2}=0.1187 \pm 0.0017$ [29]. Accordingly, we require the relic density of $X$ to satisfy the $90 \%$ CL (confidence level) range of its experimental value, $0.1159 \leq \Omega h^{2} \leq 0.1215$. As mentioned in the preceding section, for simplicity we take $g_{X}=g_{B-L}$, implying that $|\theta| \simeq R_{v}$. The plots in figure 1 display the resulting $g_{X}$ values allowed by the relic data for $R_{v}=10^{-2}$ (red solid curve) and $10^{-3}$ (blue solid curve) on the left and right panels, respectively. One can see that, although the $f \bar{f} Z_{L}$ couplings are suppressed by the small mixing angle, $|\theta| \ll 1$, the observed relic density can be reproduced with moderate-sized couplings $g_{B-L}=g_{X}=\mathcal{O}(0.1)-\mathcal{O}(1)$ over $m_{X} \leq 1000 \mathrm{GeV}$ due to the resonance enhancement. This can be partly understood from the fact that in the resonance region the denominator of $\sigma_{\text {ann }}$ is dominated by the term $\left(4 m_{X}^{2}-m_{Z_{L}}^{2}\right)^{2} \propto R_{v}^{2}$ which approximately cancels the $R_{v}^{2}$ factor in the numerator.

In figure 1, we can also compare the coupling ranges that fulfill the requirements from both the collider and relic density data. Evidently, the constraints from LEP II data restrict the allowed masses to $m_{X} \gtrsim 400(220) \mathrm{GeV}$ with couplings of $\mathcal{O}(1)$ for $R_{v}=10^{-2}\left(10^{-3}\right)$. The cases with $R_{v} \lesssim 10^{-4}$ and $m_{X} \leq 1000 \mathrm{GeV}$ are excluded by the LEP II constraints.

Since we have the relation $m_{X}=g_{X} v_{S} \sqrt{R_{v}}$ from eqs. (2.7) and (2.10), it is interesting to explore the values of $v_{S}$ subject to the same experimental requirements. We illustrate this in figure 2 obtained with the allowed $g_{X}$ ranges in figure 1 . Hence $v_{S}$ should be between about 5 and $10 \mathrm{TeV}$ in order to satisfy both the collider and relic data. This suggests that our model is compatible with the TeV-scale type-I seesaw scenario.

\section{Direct detection of dark matter}

The direct detection of $X$ relies on its scattering off a nucleon $N$ elastically, $X N \rightarrow$ $X N$, which proceeds from $Z_{L, H}$ exchanges in the $t$ channel. Since $m_{Z_{H}} \gg m_{Z_{L}}$, the $Z_{H}$ contribution can be neglected. It follows that in the nonrelativistic limit the cross section of $X N \rightarrow X N$ is

$$
\sigma_{\mathrm{el}}^{N}=\frac{g_{X}^{2} g_{B-L}^{2} \cos ^{2} \theta \sin ^{2} \theta \mu_{X N}^{2}}{\pi m_{Z_{L}}^{4}} \simeq \frac{g_{X}^{4} R_{v}^{2} \mu_{X N}^{2}}{16 \pi m_{X}^{4}},
$$

where $\mu_{X N}=m_{X} m_{N} /\left(m_{X}+m_{N}\right)$ and we have made use of $\left\langle N\left|\bar{u} \gamma^{\alpha} u+\bar{d} \gamma^{\alpha} d\right| N\right\rangle=$ $3 \bar{N} \gamma^{\alpha} N$ [30], the other quarks having vanishing contributions. This indicates that $\sigma_{\mathrm{el}}^{N}$ is not sensitive to $g_{B-L}$ for fixed $R_{v} \ll 1$.

In figure 3 we plot $\sigma_{\mathrm{el}}^{N}$ as a function of $m_{X}$ for the allowed parameter regions in figure 1, the red and blue strips belonging to the $R_{v}=10^{-2}$ and $10^{-3}$ cases, respectively. Also shown are the recent data from DM direct searches. Clearly, much of the $\sigma_{\text {el }}^{N}$ prediction 


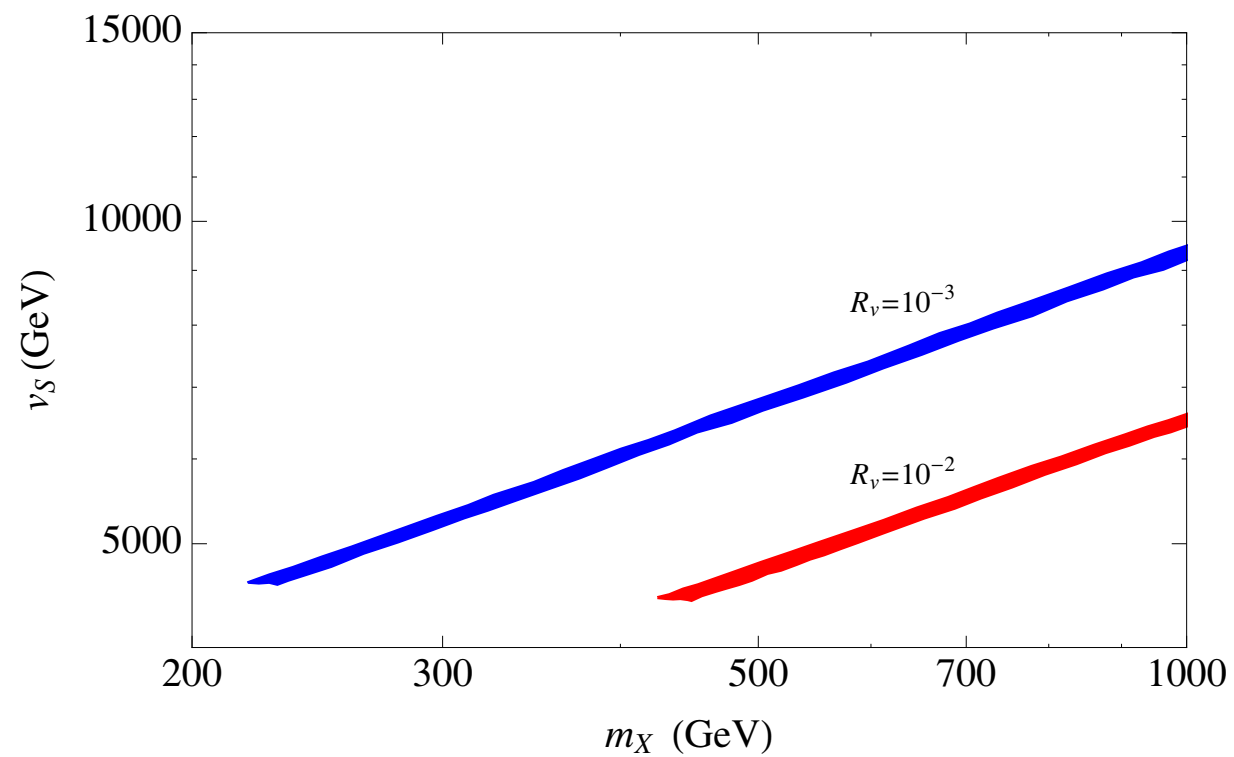

Figure 2. Values of $v_{S}$ versus $m_{X}$ satisfying the requirements from both the collider and relic density data and corresponding to the allowed $g_{X}$ regions in figure 1 .

still escapes the existing constraints, including the strictest ones from XENON100 [33] and LUX [38], but it will be probed more stringently by future direct searches such as XENON1T [46].

Before moving on, we would like to make a few remarks regarding the potential implications of mixing between the SM and extra gauge bosons in our model. Since none of the scalar fields in the theory carries both the electroweak and new quantum numbers, there is no mass mixing between the SM and new gauge bosons. In contrast, as discussed in appendix $\mathrm{B}$, kinetic mixing between the $\mathrm{U}(1)_{Y}$ and $\mathrm{U}(1)_{B-L}$ gauge bosons can occur both at tree and loop levels. We find that the impact of this mixing is not significant on the results above for the allowed values of the new gauge couplings and $Z_{L}$ mass. Especially, the relation $m_{Z_{L}} \simeq 2 m_{X}$ is unaffected. We further find that, although the $Z_{H}$ mass is sensitive to the kinetic mixing, being enhanced by it, the effect can be minimized if the mixing parameter has a magnitude below 0.5. Our rough estimate of the relevant loop diagram in appendix B suggests that mixing size of order 0.5 is not atypical. Lastly, since the $X$ annihilation and $X$-nucleon scattering processes are dominated by the $Z_{L}$ contributions, the increased $m_{Z_{H}}$ would not be important for them. It follows that it is reasonable to neglect the impact of the kinetic mixing.

\section{Comments on collider phenomenology}

In this section, we briefly discuss how the extra scalar and gauge bosons in our model may be produced and detected at the LHC. The new scalar bosons coming from $\Phi_{5}$ and $S$ comprise twelve degrees of freedom in total. Four of them are "eaten" by the new gauge bosons, making them massive. The remaining extra scalar bosons can be expressed as $\phi_{i}^{\text {even }}$ and $\phi_{j}^{\text {odd }}$, which are linear combinations of $Z_{2}^{X}$-even and -odd particles, respectively. Since 


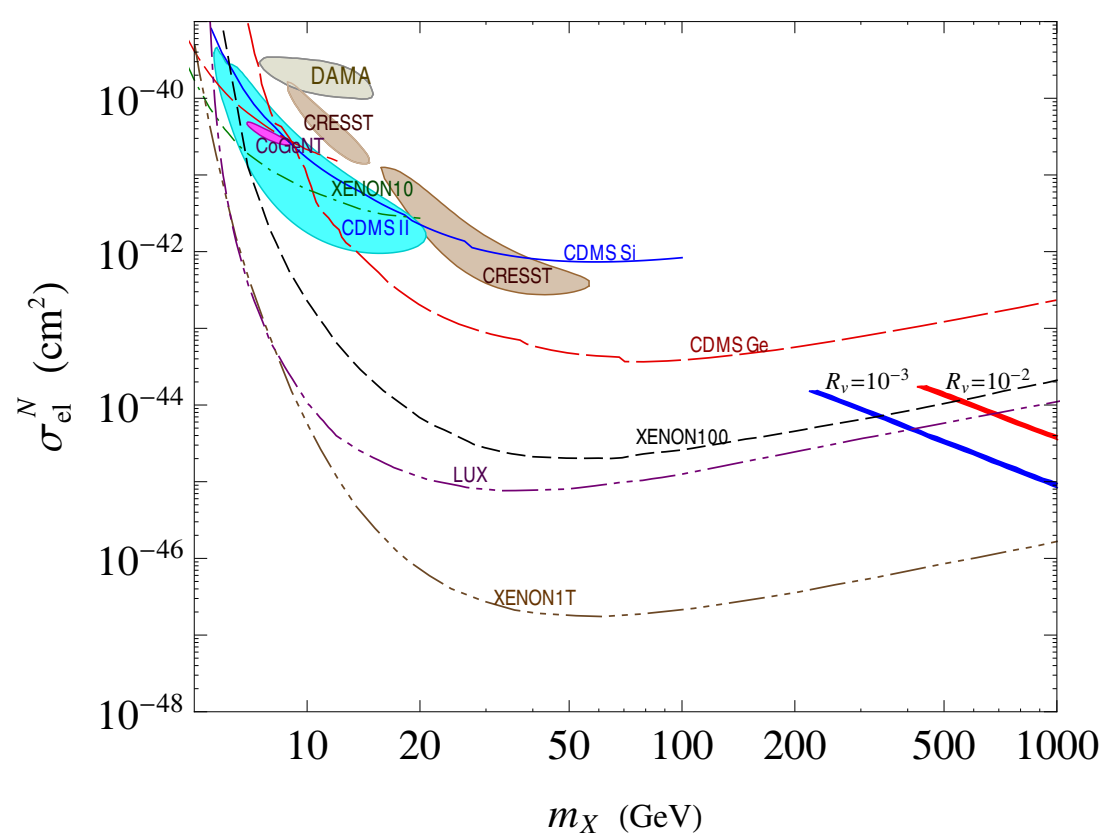

Figure 3. Cross-section $\sigma_{\mathrm{el}}^{N}$ of $X N \rightarrow X N$ scattering corresponding to the allowed parameter regions in figure 1 . The predicted cross-sections are compared to $90 \% \mathrm{CL}$ upper-limits from XENON10 (green dashed-dotted curve) [31, 32], XENON100 (black short-dashed curve) [33], CDMS Ge (red long-dashed curves) [34-36], CDMS Si (blue solid curve) [37], and LUX (purple dasheddouble-dotted curve) [38]. The prediction is also compared to the $90 \%$ CL (magenta) signal region suggested by CoGeNT [39-41], a gray patch compatible with the DAMA modulation signal at the $3 \sigma$ level [42, 43], two 2 $\sigma$-confidence (light brown) areas representing CRESST-II data [44], and a cyan area for a possible signal at 90\% CL from CDMS II [45]. Also plotted is the XENON1T projected sensitivity (brown dashed-triple-dotted curve) [46].

two of the new massive gauge bosons are $Z_{2}^{X}$ even and the other two $Z_{2}^{X}$ odd, there are six

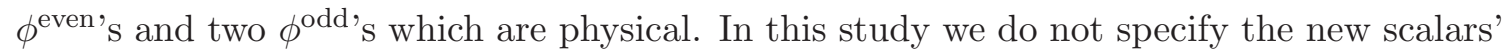
mass spectrum, but one could obtain it by doing a detailed analysis of the scalar potential.

Taking into account the $Z_{2}^{X}$ parities of the new particles, we find the decay patterns

$$
\begin{aligned}
Z_{L} & \rightarrow f_{\mathrm{SM}} \bar{f}_{\mathrm{SM}}, \\
Z_{H} & \rightarrow f_{\mathrm{SM}} \bar{f}_{\mathrm{SM}}, X X^{\dagger}, \phi_{i}^{\text {even }} \phi_{j}^{\text {even }}, \phi_{i}^{\text {odd }} \phi_{j}^{\text {odd }}, \\
\phi_{i}^{\text {even }} & \rightarrow Z_{L, H} Z_{L, H}, X X^{\dagger}, X^{(\dagger)} \phi_{i}^{\text {odd }}, Z_{L, H} \phi_{i}^{\text {even }}, \phi_{j}^{\text {even }} \phi_{k}^{\text {even }}, \phi_{j}^{\text {odd }} \phi_{k}^{\text {odd }}, \\
\phi_{i}^{\text {odd }} & \rightarrow Z_{L, H} X, \phi_{j}^{\text {even }} X, \phi_{j}^{\text {even }} \phi_{k}^{\text {odd }},
\end{aligned}
$$

where the particles on the right-hand sides may be off-shell depending on the masses involved. Throughout we have assumed that $X$ is lighter than new scalar bosons, and so $Z_{L}$ decays mostly to SM fermions. Since the couplings of $Z_{L, H}$ to the fermions are proportional to their $B-L$ numbers, $Z_{L, H}$ tend to decay into leptons rather than quarks, as the decay rates of $Z_{L, H}$ into a charged lepton pair and into a quark-antiquark pair, with relatively negligible masses, are related by $\Gamma_{Z_{L, H} \rightarrow \ell^{+} \ell^{-}}: \Gamma_{Z_{L, H} \rightarrow q \bar{q}} \simeq 1: 3(1 / 3)^{2}=3: 1$. 
The decay branching fractions of the scalar bosons depend on their mass spectrum and couplings in the potential.

The DM pair, $X X^{\dagger}$, can be produced through $Z_{L, H}$ exchanges in the $s$-channel according to

$$
p p \rightarrow Z_{L, H}^{(*)}+[\text { jet(s), photon(s), etc. }] \rightarrow X X^{\dagger}+[\text { jet(s), photon(s), etc. }],
$$

where we need particles other than $X X^{\dagger}$, such as jets ( $j$ 's) or photons $\left(\gamma^{\prime}\right.$ 's), for tagging. Their production signals are therefore jet(s) plus missing energy, photon(s) plus missing energy, etc. We note that the $Z_{L}$-mediated contributions dominate these processes because $Z_{H}$ is much heavier than $Z_{L}$.

We now estimate the cross-sections of $p p \rightarrow X X^{\dagger} j$ and $p p \rightarrow X X^{\dagger} \gamma$ using the CalcHEP code package [25] incorporating the new Feynman rules in the model file. The cross sections are computed for the Tevatron and the LHC with different colliding energies employing two parameter sets taken from figure 1 . The results are listed in table 2 . These cross sections are small compared to current search limits due to the suppression by the small $|\theta|$. For example, it is estimated that the upper limit of the cross section for jets plus missing energy in the squark-gluino-neutralino scenario of the minimal supersymmetric standard model is $\mathcal{O}(1) \mathrm{fb}$ for the LHC at $\sqrt{s}=7 \mathrm{TeV}$ with $4.7 \mathrm{fb}^{-1}$ of luminosity [47], which is larger than our cross sections. However, at the LHC the cross-section of $p p \rightarrow$ $X X^{\dagger} j$ can reach $\sim 0.1 \mathrm{fb}$ for $\sqrt{s}=8 \mathrm{TeV}$ and $\sim 0.5 \mathrm{fb}$ for $\sqrt{s}=14 \mathrm{TeV}$, which are potentially testable with the appropriate amount of luminosity. The DM can also be produced singly in association with $\phi_{i}^{\text {odd }}$, such as in

$$
p p \rightarrow Z_{L, H}^{(*)}+\cdots \rightarrow X^{(\dagger)} \phi_{i}^{\text {odd }}+\cdots .
$$

Since $\phi^{\text {odd }}$ would decay according to eq. (6.4), the specific signal would be charged leptons plus missing energy: $X^{(\dagger)} \phi_{i}^{\text {odd }} \rightarrow X X^{\dagger} Z_{L} \rightarrow X X^{\dagger} \ell^{+} \ell^{-}$where $\ell$ is the electron or muon. The cross section of this channel is expected to be of similar order as that of $X X^{\dagger}$ production since the coupling constants involved are the same.

The scalar bosons $\phi_{i}^{\text {even }}$ and $\phi_{i}^{\text {odd }}$ can be produced through $Z_{L, H}$ exchanges in the $s$ channel,

$$
\begin{aligned}
& p p \rightarrow Z_{L, H}^{*} \rightarrow Z_{L, H} \phi_{i}^{\text {even }}, \\
& p p \rightarrow Z_{L, H}^{(*)} \rightarrow \phi_{i}^{\text {odd }} \phi_{j}^{\text {odd }}, \\
& p p \rightarrow Z_{L, H}^{(*)} \rightarrow \phi_{i}^{\text {even }} \phi_{j}^{\text {even }},
\end{aligned}
$$

as well as the channels in eq. (6.6). According to the decay patterns in eqs. (6.3) and (6.4), the signals of $\phi_{i}^{\text {even }}$ and $\phi_{i}^{\text {odd }}$ would be two pairs of charged leptons and charged leptons plus missing energy, respectively. The production cross-sections of the $Z_{L^{-}}$exchange processes are suppressed by $|\theta|^{2}$ because the $Z_{L} f \bar{f}$ coupling is proportional to $\sin \theta$, whereas the production cross-sections of $Z_{H}$-exchange processes are suppressed by the heavy $Z_{H}$ mass. Thus high luminosities will be required to get a sufficient number of signal events. To carry out a detailed analysis, one would need to specify the self-couplings and thus the mass spectrum of scalar bosons. We leave such a study for future work. 


\begin{tabular}{|l|c|c|c|c|c|}
\hline & $m_{X}(\mathrm{GeV})$ & $g_{X}$ & $|\theta|$ & $\sigma_{X X^{\dagger} j}(\mathrm{fb})$ & $\sigma_{X X^{\dagger} \gamma}(\mathrm{fb})$ \\
\hline Tevatron & 300 & 1.8 & 0.001 & $6.5 \times 10^{-3}$ & $1.7 \times 10^{-4}$ \\
& 600 & 1.2 & 0.01 & $6.0 \times 10^{-5}$ & $1.8 \times 10^{-6}$ \\
\hline LHC $7 \mathrm{TeV}$ & 300 & 1.8 & 0.001 & $8.7 \times 10^{-2}$ & $1.6 \times 10^{-3}$ \\
& 600 & 1.2 & 0.01 & $5.1 \times 10^{-2}$ & $1.1 \times 10^{-3}$ \\
\hline LHC $8 \mathrm{TeV}$ & 300 & 1.8 & 0.001 & $8.6 \times 10^{-2}$ & $2.3 \times 10^{-3}$ \\
& 600 & 1.2 & 0.01 & 0.12 & $1.9 \times 10^{-3}$ \\
\hline LHC $14 \mathrm{TeV}$ & 300 & 1.8 & 0.001 & 0.46 & $8.2 \times 10^{-3}$ \\
& 600 & 1.2 & 0.01 & 0.51 & $1.0 \times 10^{-2}$ \\
\hline
\end{tabular}

Table 2. Estimated cross-sections of $p p(p \bar{p}) \rightarrow X X^{\dagger} j$ and $p p(p \bar{p}) \rightarrow X X^{\dagger} \gamma$ for two parameter sets taken from figure 1.

\section{Summary and discussion}

In this paper we have constructed a model possessing an extra gauge symmetry, $\mathrm{SU}(2)_{X} \times \mathrm{U}(1)_{B-L}$, which offers a massive gauge boson, $X$, associated with $\mathrm{SU}(2)_{X}$ playing the role of WIMP DM. The new gauge bosons become massive after $\mathrm{SU}(2)_{X}$ and $\mathrm{U}(1)_{B-L}$ are spontaneously broken by scalar fields $\Phi_{5}$ and $S$ developing nonzero VEVs of $v_{\Phi}$ and $v_{S}$, respectively, with $v_{S} \gg v_{\Phi}$. The stability of the DM candidate is guaranteed by a residual $Z_{2}$ symmetry that is a subgroup of $\mathrm{SU}(2)_{X}$. At tree level, the dark gauge boson $X$ can interact with SM fermions by exchanging new gauge bosons $Z_{L, H}$ which arise from linear combinations of $\mathrm{SU}(2)_{X}$ and $\mathrm{U}(1)_{B-L}$ gauge fields. The $X X^{\dagger}$ pair annihilates into SM fermions by exchanging $Z_{L, H}$ in the $s$ channel. Since the DM is a gauge boson, its mass can be related to the masses of other gauge bosons in the model. The relation $m_{Z_{L}} \simeq 2 m_{X}$ emerges when $\mathrm{SU}(2)_{X}$ is broken by the $\mathrm{VEV}$ of the $\mathrm{SU}(2)_{X}$ scalar five-plet $\Phi_{5}$, naturally leading to resonant pair annihilation of $X X^{\dagger}$ via $Z_{L}$ exchange. This model also supplies light neutrino masses with the aid of right-handed neutrinos whose mass terms are generated when $\mathrm{U}(1)_{B-L}$ is spontaneously broken by the VEV of $S$, which activates the type-I seesaw mechanism.

We considered the values of the new gauge couplings $g_{X}$ and $g_{B-L}$ in the case where they are equal subject to constraints from collider data and the observed relic density. Assuming that $R_{v}=v_{\Phi}^{2} / v_{S}^{2} \ll 1$ and $m_{X} \leq 1 \mathrm{TeV}$, we obtain no parameter space which survives these experimental restrictions for $R_{v} \lesssim 10^{-4}$, but the $m_{X} \gtrsim 400(220) \mathrm{GeV}$ region with $\mathcal{O}(1)$ couplings is still allowed for $R_{v}=10^{-2}\left(10^{-3}\right)$. We also find that the corresponding values of $v_{S}$ are between 5 and $10 \mathrm{TeV}$, implying that our model prefers the TeV-scale type-I seesaw scenario. Subsequently, we explored the $X$-nucleon elastic scattering cross-section, $\sigma_{\mathrm{el}}^{N}$, for the surviving parameter regions and compared it with data from the latest DM direct detection experiments. The resulting $\sigma_{\mathrm{el}}^{N}$ largely evades the most recent XENON100 and LUX limits and will be tested more strictly by future DM direct searches such as XENON1T. 
Finally, we schematically discussed some of the phenomenology of the new particles at the LHC. The DM particle can be produced as $X X^{\dagger}$ or $X^{(\dagger)} \phi_{i}^{\text {odd }}$, where $\phi_{i}^{\text {odd }}$ is a $Z_{2}^{X}$-odd scalar boson. The signals of these production processes would be missing energy plus jets/photons and missing energy plus charged lepton pairs, respectively. The new scalar bosons can also be produced as $\phi^{\text {even(odd) }} \phi^{\text {even(odd) }}$ or $\phi^{\text {odd }} X\left(\phi^{\text {even }} Z_{L, H}\right)$, and the signals for $\phi^{\text {even }}$ and $\phi^{\text {odd }}$ would be two pairs of charged leptons and missing energy plus charged leptons, respectively. Their production cross-sections tend to be suppressed due to the small $|\theta|$ value and/or the heavy $Z_{H}$ mass. Nevertheless, our model would be testable with an appropriate luminosity in the future. To perform a more detailed study would require specifying the self-couplings in the scalar potential and thus the mass spectrum of the scalar bosons.

\section{Acknowledgments}

This research was supported in part by the National Science Council of Taiwan, R. O. C., under Grants No. NSC-100-2628-M-008-003-MY4 and No. NSC-100-2811-M-002-090 and by the MOE Academic Excellence Program under Grant No. 102R891505.

\section{A Feynman rules for new gauge interactions}

The couplings of the fermion $f$ in the model with the $Z_{L, H}$ bosons come from the $\mathrm{U}(1)_{B-L}$ gauge interaction of $f$ described by

$$
\mathcal{L}^{\prime}=-g_{B-L} \bar{f} \gamma^{\mu}\left(\hat{L}_{f}^{E} P_{L}+\hat{R}_{f}^{E} P_{R}\right) f E_{\mu},
$$

where $P_{L, R}=\frac{1}{2}\left(1 \mp \gamma_{5}\right)$ and $\hat{L}_{f}^{E}\left(\hat{R}_{f}^{E}\right)$ is the $B-L$ value for $f_{L(R)}$. Since $E=Z_{L} \sin \theta+$ $Z_{H} \cos \theta$, this leads to the Feynman rules

$$
\begin{aligned}
& \bar{f} f Z_{L}^{\mu}:-i g_{B-L} \sin \theta\left(\hat{V}_{f}^{E}+\hat{A}_{f}^{E} \gamma_{5}\right) \gamma^{\mu}, \\
& \bar{f} f Z_{H}^{\mu}:-i g_{B-L} \cos \theta\left(\hat{V}_{f}^{E}+\hat{A}_{f}^{E} \gamma_{5}\right) \gamma^{\mu},
\end{aligned}
$$

where $2 \hat{V}_{f}^{E}=\hat{L}_{f}^{E}+\hat{R}_{f}^{E}$ and $2 \hat{A}_{f}^{E}=\hat{L}_{f}^{E}-\hat{R}_{f}^{E}$. From the kinetic term of the $C_{k}$ gauge bosons, $-\frac{1}{4} C_{k \mu \nu} C_{k}^{\mu \nu}$, where $C_{k}^{\mu \nu}=\partial^{\mu} C_{k}^{\nu}-\partial^{\nu} C_{k}^{\mu}-g_{X} \epsilon_{k r s} C_{r}^{\mu} C_{s}^{\nu}$, with $C_{3}=Z_{L} \cos \theta-$ $Z_{H} \sin \theta$, we derive the Feynman rules

$$
\begin{aligned}
& X^{\mu} X^{\nu \dagger} Z_{L}^{\rho}:-i g_{X} \cos \theta\left[\left(p_{X}^{\rho}-p_{X^{\dagger}}^{\rho}\right) g^{\mu \nu}+\left(p_{X^{\dagger}}^{\mu}-p_{Z_{L}}^{\mu}\right) g^{\nu \rho}+\left(p_{Z_{L}}^{\nu}-p_{X}^{\nu}\right) g^{\mu \rho}\right], \\
& X^{\mu} X^{\nu \dagger} Z_{H}^{\rho}:-i g_{X} \sin \theta\left[\left(p_{X}^{\rho}-p_{X^{\dagger}}^{\rho}\right) g^{\mu \nu}+\left(p_{X^{\dagger}}^{\mu}-p_{Z_{H}}^{\mu}\right) g^{\nu \rho}+\left(p_{Z_{H}}^{\nu}-p_{X}^{\nu}\right) g^{\mu \rho}\right], \\
& X_{\mu} X_{\nu} X_{\rho}^{\dagger} X_{\sigma}^{\dagger}: i g_{X}^{2}\left(g_{\mu \nu} g_{\rho \sigma}-g_{\mu \rho} g_{\nu \sigma}\right), \\
& X_{\mu} X_{\nu}^{\dagger} Z_{L \rho} Z_{L \sigma}: i g_{X}^{2} \cos ^{2} \theta\left(g_{\mu \nu} g_{\rho \sigma}-g_{\mu \rho} g_{\nu \sigma}\right), \\
& X_{\mu} X_{\nu}^{\dagger} Z_{H \rho} Z_{H \sigma}: i g_{X}^{2} \sin ^{2} \theta\left(g_{\mu \nu} g_{\rho \sigma}-g_{\mu \rho} g_{\nu \sigma}\right), \\
& X_{\mu} X_{\nu}^{\dagger} Z_{L \rho} Z_{H \sigma}: 2 i g_{X}^{2} \sin \theta \cos \theta\left(g_{\mu \nu} g_{\rho \sigma}-g_{\mu \rho} g_{\nu \sigma}\right),
\end{aligned}
$$

where the momenta are all incoming. 


\section{B Kinetic mixing between $\mathrm{U}(1)_{Y}$ and $\mathrm{U}(1)_{B-L}$}

In the gauge $\mathrm{SU}(2)_{L} \times \mathrm{U}(1)_{Y} \times \mathrm{SU}(2)_{X} \times \mathrm{U}(1)_{B-L}$ sector of our model, the gauge bosons that may undergo mixing are $W_{3}, \mathcal{B}, C_{3}$, and $E$, respectively. We can express the Lagrangian for the kinetic and mass terms of these particles after electroweak symmetry breaking as

$$
\begin{aligned}
\mathcal{L}_{G}= & -\frac{1}{4} W_{3}^{\alpha \omega} W_{3 \alpha \omega}-\frac{1}{4} \mathcal{B}^{\alpha \omega} \mathcal{B}_{\alpha \omega}-\frac{1}{4} C_{3}^{\alpha \omega} C_{3 \alpha \omega}-\frac{1}{4} E^{\alpha \omega} E_{\alpha \omega}-\frac{1}{2} \sin \chi \mathcal{B}^{\alpha \omega} E_{\alpha \omega} \\
& +\frac{1}{2} m_{W}^{2} W_{3}^{2}+\frac{1}{2} m_{\mathcal{B}}^{2} \mathcal{B}^{2}-m_{W} m_{\mathcal{B}} W_{3}^{\alpha} \mathcal{B}_{\alpha}+\frac{1}{2} m_{C_{3}}^{2} C_{3}^{2}+\frac{1}{2} m_{E}^{2} E^{2}+m_{C_{3}} \mu_{E} C_{3}^{\alpha} E_{\alpha} \\
= & -\frac{1}{4} G_{\alpha \omega}^{\mathrm{T}} K G^{\alpha \omega}+\frac{1}{2} G_{\alpha}^{\mathrm{T}} M_{G}^{2} G^{\alpha}
\end{aligned}
$$

where $f_{\alpha \omega}=\partial_{\alpha} f_{\omega}-\partial_{\omega} f_{\alpha}$, the $\sin \chi$ term describes kinetic mixing between the hypercharge and $B-L$ gauge bosons, $\mathcal{B}$ and $E$, respectively,

$$
\begin{array}{rlrl}
m_{W} & =\frac{1}{2} g_{L} v_{H}, \quad m_{\mathcal{B}}=\frac{1}{2} g_{Y} v_{H}, \\
m_{C_{3}} & =2 g_{X} v_{\Phi}, \quad m_{E}^{2}=4 g_{B-L}^{2} v_{S}^{2}+\mu_{E}^{2}, \quad \mu_{E}=2 g_{B-L} v_{\Phi}, \\
G & =\left(\begin{array}{c}
\mathcal{B} \\
W_{3} \\
E \\
C_{3}
\end{array}\right), \quad K=\left(\begin{array}{cccc}
1 & 0 & s_{\chi} & 0 \\
0 & 1 & 0 & 0 \\
s_{\chi} & 0 & 1 & 0 \\
0 & 0 & 0 & 1
\end{array}\right), & M_{G}^{2}=\left(\begin{array}{cccc}
m_{\mathcal{B}}^{2} & -m_{\mathcal{B}} m_{W} & 0 & 0 \\
-m_{\mathcal{B}} m_{W} & m_{W}^{2} & 0 & 0 \\
0 & 0 & m_{E}^{2} & \mu_{E} m_{C_{3}} \\
0 & 0 & \mu_{E} m_{C_{3}} & m_{C_{3}}^{2}
\end{array}\right),
\end{array}
$$

with $s_{\chi}=\sin \chi$ and $g_{L}$ and $g_{Y}$ being the $\mathrm{SU}(2)_{L} \times \mathrm{U}(1)_{Y}$ gauge couplings, respectively. In $\mathcal{L}_{G}$ we have included the $s_{\chi}$ term because it does not violate any of the symmetries in the theory, implying that in general $s_{\chi}$ can receive both tree- and loop-level contributions [4952 , the latter being due to the $\mathrm{SM}$ fermions carrying both the $\mathrm{U}(1)_{Y}$ and $\mathrm{U}(1)_{B-L}$ charges.

It is straightforward to demonstrate that one can convert the kinetic part of $\mathcal{L}_{G}$ into the canonical form, $-\frac{1}{4} \hat{G}_{\alpha \omega}^{\mathrm{T}} \hat{G}^{\alpha \omega}$, and diagonalize the $M_{G}^{2}$ matrix by making the transformation

$$
G=\tilde{T} O_{\mathrm{w}} \mathcal{O}_{z}\left(\begin{array}{c}
A \\
Z \\
Z_{H} \\
Z_{L}
\end{array}\right)
$$

where $A, Z, Z_{H}$, and $Z_{L}$ are the mass eigenstates, the photon $A$ staying massless,

$$
\begin{array}{rlrl}
\tilde{T} & =\left(\begin{array}{cccc}
1 & 0 & -t_{\chi} & 0 \\
0 & 1 & 0 & 0 \\
0 & 0 & 1 / c_{\chi} & 0 \\
0 & 0 & 0 & 1
\end{array}\right), & O_{\mathrm{w}}=\left(\begin{array}{cccc}
c_{\mathrm{w}} & -s_{\mathrm{w}} & 0 & 0 \\
s_{\mathrm{w}} & c_{\mathrm{w}} & 0 & 0 \\
0 & 0 & 1 & 0 \\
0 & 0 & 0 & 1
\end{array}\right), & \mathcal{O}_{z}=\left(\begin{array}{cccc}
1 & 0 & 0 & 0 \\
0 & \mathcal{O}_{11} & \mathcal{O}_{12} & \mathcal{O}_{13} \\
0 & \mathcal{O}_{21} & \mathcal{O}_{22} & \mathcal{O}_{23} \\
0 & \mathcal{O}_{31} & \mathcal{O}_{32} & \mathcal{O}_{33}
\end{array}\right), \\
c_{\chi}=\cos \chi, & t_{\chi}=\tan \chi, & c_{\mathrm{w}}=\cos \theta_{\mathrm{W}} \\
s_{\mathrm{w}} & =\sin \theta_{\mathrm{W}}=\frac{c_{\mathrm{w}} m_{\mathcal{B}}}{m_{W}} &
\end{array}
$$


The $O_{\mathrm{w}}$ and $\mathcal{O}_{z}$ matrices are orthogonal, while $\tilde{T}$ is not. The elements $\mathcal{O}_{i j}$ encode the effect of the kinetic mixing, such that in its absence they are given by $\mathcal{O}_{11}=1, \mathcal{O}_{12}=$ $\mathcal{O}_{21}=\mathcal{O}_{13}=\mathcal{O}_{31}=0, \mathcal{O}_{22}=\mathcal{O}_{33}=\cos \theta$, and $\mathcal{O}_{23}=-\mathcal{O}_{32}=\sin \theta$, which is the case treated in the main text.

It is also simple to see that the presence of kinetic mixing, $s_{\chi} \neq 0$, affects all of the couplings of $Z$ and $Z_{L, H}$ to fermions. Since the fermions do not couple directly to the $C_{3}$ gauge boson, one can write the Lagrangian for their interactions with the $W_{3}, \mathcal{B}$, and $E$ bosons in terms of the physical states $A, Z$, and $Z_{H, L}$ as

$$
\begin{aligned}
\mathcal{L}^{\prime}= & -g_{L} J_{3}^{\lambda} W_{3 \lambda}-g_{Y} J_{Y}^{\lambda} \mathcal{B}_{\lambda}-g_{B-L} J_{B-L}^{\lambda} E_{\lambda} \\
= & -e J_{\mathrm{em}}^{\lambda} A_{\lambda}-\left[\left(\mathcal{O}_{11}+\mathcal{O}_{21} t_{\chi} s_{\mathrm{w}}\right) \hat{g}_{Z} \hat{J}_{Z}^{\lambda}+\frac{\mathcal{O}_{21}}{c_{\chi}} g_{B-L} J_{B-L}^{\lambda}-\mathcal{O}_{21} t_{\chi} c_{\mathrm{w}} e J_{\mathrm{em}}^{\lambda}\right] Z_{\lambda} \\
& -\left[\frac{\mathcal{O}_{22}}{c_{\chi}} g_{B-L} J_{B-L}^{\lambda}+\left(\mathcal{O}_{12}+\mathcal{O}_{22} t_{\chi} s_{\mathrm{w}}\right) \hat{g}_{Z} \hat{J}_{Z}^{\lambda}-\mathcal{O}_{22} t_{\chi} c_{\mathrm{w}} e J_{\mathrm{em}}^{\lambda}\right] Z_{H \lambda} \\
& -\left[\frac{\mathcal{O}_{23}}{c_{\chi}} g_{B-L} J_{B-L}^{\lambda}+\left(\mathcal{O}_{13}+\mathcal{O}_{23} t_{\chi} s_{\mathrm{w}}\right) \hat{g}_{Z} \hat{J}_{Z}^{\lambda}-\mathcal{O}_{23} t_{\chi} c_{\mathrm{w}} e J_{\mathrm{em}}^{\lambda}\right] Z_{L \lambda}
\end{aligned}
$$

where $J_{3, Y, B-L}$ are the currents coupled to the respective fields and we have used the relations

$$
e=g_{L} s_{\mathrm{w}}=g_{Y} c_{\mathrm{w}}, \quad J_{\mathrm{em}}=J_{3}+J_{Y}, \quad \hat{g}_{Z} \hat{J}_{Z}=c_{\mathrm{w}} g_{L} J_{3}-s_{\mathrm{w}} g_{Y} J_{Y} .
$$

From the previous paragraphs, one can infer that the $\chi$-dependent new terms translate into modifications to $Z$-pole observables and the $e^{+} e^{-} \rightarrow f \bar{f}$ cross-sections, as well as the $Z$ and $Z_{H, L}$ masses. Consequently, such contributions must respect the pertinent experimental restrictions. After imposing them, we find that for the ranges of the new gauge couplings and $Z_{L}$ mass satisfying the relic data requirement the kinetic-mixing effects are unimportant on the $Z$ and $Z_{L}$ masses, but for $\sin \chi$ not much less than 1 they could enlarge the $Z_{H}$ mass substantially compared to that in the $\chi=0$ case. Specifically, the increase in $m_{Z_{H}}$ would be mild, no more than about $15 \%$, if $|\sin \chi| \lesssim 0.5$.

To see if such mixing size is reasonable, we consider the two-point polarization diagram for the $\mathcal{B}$ and $E$ gauge bosons with fermions in the loop. Accordingly, we estimate the kinetic mixing parameter to be $[49,53]$

$$
\sin \chi \simeq-\sum_{f} \frac{g_{B-L} g_{Y}}{24 \pi^{2}}\left(B_{f}-L_{f}\right) Y_{f} \ln \frac{\left|q^{2}\right|}{\Lambda^{2}},
$$

where the sum is over the SM chiral fermions, $B_{f}-L_{f}$ and $Y_{f}$ denote the $B-L$ and hypercharge values for fermion $f$, respectively, $q$ is the renormalization scale which we take to be the $\mathrm{U}(1)_{B-L}$ breaking scale, of order $1 \mathrm{TeV}$ or greater, and we have applied the renormalization condition that at some higher scale $\Lambda$ the sum of the loop and counterterm contributions vanishes. We note that one could evaluate $\sin \chi$ more precisely using the renormalization group equation to resum the large logarithms [53, 54], but the difference would amount to only a few percent for our scales of interest and therefore can be ignored. Thus, since $\sum_{f}\left(B_{f}-L_{f}\right) Y_{f}=8$, taking $\Lambda^{2} \sim 10^{6}\left|q^{2}\right|$ in eq. (B.10) we get $\sin \chi \sim$ 
$0.16 g_{B-L}$. For the viable $g_{B-L}$ values we have obtained, this result is compatible with the $|\sin \chi|$ number quoted in the last paragraph.

We remark that this is also consistent with the findings of a detailed analysis in ref. [55] on the phenomenological constraints on a new massive Abelian gauge boson. The effects of such a particle can be compared to those of $Z_{H}$ which contains mostly its $\mathrm{U}(1)_{B-L}$ component $E$ and has a mass of $\mathcal{O}(1-10) \mathrm{TeV}$ in our study. For a new massive Abelian gauge boson in this mass range, the results of ref. [55] imply $|\sin \chi| \lesssim 0.6-1$. They also do not lead to additional restraints on the corresponding viable values of $g_{B-L}$.

Open Access. This article is distributed under the terms of the Creative Commons Attribution License (CC-BY 4.0), which permits any use, distribution and reproduction in any medium, provided the original author(s) and source are credited.

\section{References}

[1] Particle Data Group collaboration, J. Beringer et al., Review of particle physics, Phys. Rev. D 86 (2012) 010001 [INSPIRE].

[2] C.W. Chiang, T. Nomura and J. Tandean, Dark matter and Higgs boson in a model with discrete gauge symmetry, Phys. Rev. D 87 (2013) 073004 [arXiv:1205.6416] [INSPIRE].

[3] L.M. Krauss and F. Wilczek, Discrete gauge symmetry in continuum theories, Phys. Rev. Lett. 62 (1989) 1221 [INSPIRE].

[4] P. Minkowski, $\mu \rightarrow$ ey at a rate of one out of 1-billion muon decays?, Phys. Lett. B 67 (1977) 421 [INSPIRE].

[5] T. Yanagida, Horizontal symmetry and masses of neutrinos, in the proceedings of the Workshop on unified theory and baryon number in the universe, O. Sawada and A. Sugamoto eds., KEK, Tsukuba, Japan (1979).

[6] T. Yanagida, Horizontal Symmetry and Masses of Neutrinos, Prog. Theor. Phys. 64 (1980) 1103 [InSPIRE].

[7] M. Gell-Mann, P. Ramond and R. Slansky, Complex spinors and unified theories, in Supergravity, D.Z. Freedman and P.van Nieuwenhuizen eds., North Holland, Amsterdam, The Netherlands (1979).

[8] S.L. Glashow, The future of elementary particle physics, in Quarks and leptons, Cargèse lectures, M. Lévy et al. eds., Plenum Press, New York, U.S.A. (1980).

[9] R.N. Mohapatra and G. Senjanović, Neutrino mass and spontaneous parity violation, Phys. Rev. Lett. 44 (1980) 912 [InSPIRE].

[10] J. Schechter and J.W.F. Valle, Neutrino masses in $\mathrm{SU}(2) \times \mathrm{U}(1)$ theories, Phys. Rev. D 22 (1980) 2227 [INSPIRE].

[11] J. Schechter and J.W.F. Valle, Neutrino decay and spontaneous violation of lepton number, Phys. Rev. D 25 (1982) 774 [INSPIRE].

[12] T. Hambye, Hidden vector dark matter, JHEP 01 (2009) 028 [arXiv:0811.0172] [INSPIRE].

[13] T. Hambye and M.H.G. Tytgat, Confined hidden vector dark matter, Phys. Lett. B 683 (2010) 39 [arXiv:0907.1007] [INSPIRE]. 
[14] F. Chen, J.M. Cline and A.R. Frey, Nonabelian dark matter: models and constraints, Phys. Rev. D 80 (2009) 083516 [arXiv:0907.4746] [InSPIRE].

[15] J.L. Diaz-Cruz and E. Ma, Neutral SU(2) gauge extension of the standard model and a vector-boson dark-matter candidate, Phys. Lett. B 695 (2011) 264 [arXiv:1007.2631] [INSPIRE].

[16] S. Bhattacharya, J.L. Diaz-Cruz, E. Ma and D. Wegman, Dark vector-gauge-boson model, Phys. Rev. D 85 (2012) 055008 [arXiv:1107.2093] [inSPIRE].

[17] O. Lebedev, H.M. Lee and Y. Mambrini, Vector Higgs-portal dark matter and the invisible Higgs, Phys. Lett. B 707 (2012) 570 [arXiv:1111.4482] [INSPIRE].

[18] Y. Farzan and A.R. Akbarieh, VDM: A model for Vector Dark Matter, JCAP 10 (2012) 026 [arXiv: 1207.4272] [INSPIRE].

[19] T. Abe, M. Kakizaki, S. Matsumoto and O. Seto, Vector WIMP miracle, Phys. Lett. B 713 (2012) 211 [arXiv:1202.5902] [InSPIRE].

[20] F. D'Eramo, M. McCullough and J. Thaler, Multiple gamma lines from semi-annihilation, JCAP 04 (2013) 030 [arXiv:1210.7817] [INSPIRE].

[21] ALEPH, DELPHI, L3, OPAL, LEP Electroweak Working Group collaboration, J. Alcaraz et al., A combination of preliminary electroweak measurements and constraints on the standard model, hep-ex/0612034 [INSPIRE].

[22] C.-W. Chiang, Y.-F. Lin and J. Tandean, Probing leptonic interactions of a family-nonuniversal $Z^{\prime}$ boson, JHEP 11 (2011) 083 [arXiv:1108.3969] [INSPIRE].

[23] CMS Collaboration, Measurement of the differential and double-differential Drell-Yan cross-section in proton-proton collisions at $7 \mathrm{TeV}$, CMS-PAS-EWK-11-007 (2011).

[24] C.-W. Chiang, N.D. Christensen, G.-J. Ding and T. Han, Discovery in Drell-Yan processes at the LHC, Phys. Rev. D 85 (2012) 015023 [arXiv:1107.5830] [INSPIRE].

[25] A. Pukhov et al., CompHEP: a package for evaluation of Feynman diagrams and integration over multiparticle phase space, hep-ph/9908288 [INSPIRE].

[26] E.W. Kolb and M. Turner, The early universe, Westview Press, Boulder, U.S.A. (1990).

[27] K. Griest and D. Seckel, Three exceptions in the calculation of relic abundances, Phys. Rev. D 43 (1991) 3191 [INSPIRE].

[28] P. Gondolo and G. Gelmini, Cosmic abundances of stable particles: improved analysis, Nucl. Phys. B 360 (1991) 145 [InSPIRE].

[29] Planck collaboration, P. Ade et al., Planck 2013 results. I. Overview of products and scientific results, arXiv:1303.5062 [INSPIRE].

[30] D.B. Kaplan and A. Manohar, Strange matrix elements in the proton from neutral current experiments, Nucl. Phys. B 310 (1988) 527 [InSPIRE].

[31] XENON10 collaboration, J. Angle et al., A search for light dark matter in XENON10 data, Phys. Rev. Lett. 107 (2011) 051301 [arXiv: 1104.3088] [INSPIRE].

[32] M.T. Frandsen, F. Kahlhoefer, C. McCabe, S. Sarkar and K. Schmidt-Hoberg, The unbearable lightness of being: CDMS versus XENON, JCAP 07 (2013) 023 [arXiv: 1304.6066] [INSPIRE]. 
[33] XENON100 collaboration, E. Aprile et al., Dark matter results from 225 live days of XENON100 data, Phys. Rev. Lett. 109 (2012) 181301 [arXiv:1207.5988] [INSPIRE].

[34] CDMS-II collaboration, Z. Ahmed et al., Dark matter search results from the CDMS II experiment, Science 327 (2010) 1619 [arXiv: 0912.3592] [INSPIRE].

[35] CDMS collaboration, D. Akerib et al., A low-threshold analysis of CDMS shallow-site data, Phys. Rev. D 82 (2010) 122004 [arXiv: 1010.4290] [INSPIRE].

[36] CDMS-II collaboration, Z. Ahmed et al., Results from a low-energy analysis of the CDMS II germanium data, Phys. Rev. Lett. 106 (2011) 131302 [arXiv:1011.2482] [INSPIRE].

[37] CDMS collaboration, R. Agnese et al., Silicon detector results from the first five-tower run of CDMS II, Phys. Rev. D 88 (2013) 031104 [arXiv:1304.3706] [InSPIRE].

[38] LUX collaboration, D. Akerib et al., First results from the LUX dark matter experiment at the Sanford Underground Research Facility, arXiv:1310.8214 [INSPIRE].

[39] CoGeNT collaboration, C. Aalseth et al., CoGeNT: a search for low-mass dark matter using p-type point contact germanium detectors, Phys. Rev. D 88 (2013) 012002 [arXiv: 1208.5737] [INSPIRE].

[40] CoGeNT collaboration, C. Aalseth et al., Results from a search for light-mass dark matter with a p-type point contact germanium detector, Phys. Rev. Lett. 106 (2011) 131301 [arXiv: 1002.4703] [INSPIRE].

[41] C. Aalseth et al., Search for an annual modulation in a p-type point contact germanium dark matter detector, Phys. Rev. Lett. 107 (2011) 141301 [arXiv:1106.0650] [InSPIRE].

[42] C. Savage, G. Gelmini, P. Gondolo and K. Freese, Compatibility of DAMA/LIBRA dark matter detection with other searches, JCAP 04 (2009) 010 [arXiv:0808.3607] [INSPIRE].

[43] DAMA, LIBRA collaboration, R. Bernabei et al., New results from DAMA/LIBRA, Eur. Phys. J. C 67 (2010) 39 [arXiv:1002.1028] [InSPIRE].

[44] G. Angloher et al., Results from $730 \mathrm{~kg}$ days of the CRESST-II dark matter search, Eur. Phys. J. C 72 (2012) 1971 [arXiv:1109.0702] [INSPIRE].

[45] CDMS collaboration, R. Agnese et al., Silicon detector dark matter results from the final exposure of CDMS II, arXiv:1304.4279 [INSPIRE].

[46] XENON1T collaboration, E. Aprile, The XENON1T dark matter search experiment, arXiv: 1206.6288 [INSPIRE].

[47] ATLAS collaboration, Search for squarks and gluinos with the ATLAS detector in final states with jets and missing transverse momentum using $4.7 \mathrm{fb}^{-1}$ of $\sqrt{\mathrm{s}}=7 \mathrm{TeV}$ proton-proton collision data, Phys. Rev. D 87 (2013) 012008 [arXiv:1208.0949] [InSPIRE].

[48] G. Steigman, B. Dasgupta and J.F. Beacom, Precise relic WIMP abundance and its impact on searches for dark matter annihilation, Phys. Rev. D 86 (2012) 023506 [arXiv:1204.3622] [INSPIRE].

[49] B. Holdom, Two U(1)'s and epsilon charge shifts, Phys. Lett. B 166 (1986) 196 [InSPIRE].

[50] F. del Aguila, G.D. Coughlan and M. Quirós, Gauge coupling renormalization with several U(1) factors, Nucl. Phys. B 307 (1988) 633 [Erratum ibid. B 312 (1989) 751] [INSPIRE].

[51] R. Foot and X.-G. He, Comment on ZZ' mixing in extended gauge theories, Phys. Lett. B 267 (1991) 509 [INSPIRE]. 
[52] R. Foot, X. He, H. Lew and R. Volkas, Model for a light $Z^{\prime}$ boson, Phys. Rev. D 50 (1994) 4571 [hep-ph/9401250] [INSPIRE].

[53] K.R. Dienes, C.F. Kolda and J. March-Russell, Kinetic mixing and the supersymmetric gauge hierarchy, Nucl. Phys. B 492 (1997) 104 [hep-ph/9610479] [INSPIRE].

[54] K. Babu, C.F. Kolda and J. March-Russell, Leptophobic U(1) s and the R(b)-R(c) crisis, Phys. Rev. D 54 (1996) 4635 [hep-ph/9603212] [INSPIRE].

[55] M. Williams, C. Burgess, A. Maharana and F. Quevedo, New constraints (and motivations) for abelian gauge bosons in the MeV-TeV mass range, JHEP 08 (2011) 106 [arXiv: 1103.4556] [INSPIRE]. 